\title{
Stellenwert der Insulinanaloga bei der Therapie von Menschen mit Typ-2-Diabetes
}

\author{
Significance of insulin analogues in the treatment of people with \\ type 2 diabetes
}

Autoren

Institut

\section{B. Gallwitz ${ }^{1}$ D. Westrup ${ }^{2}$ G.-W. Schmeisl ${ }^{3}$}

\author{
${ }^{1}$ Medizinische Klinik IV, Universitätsklinikum Tübingen \\ 2 Novo Nordisk Pharma GmbH, Mainz \\ ${ }^{3}$ Deegenbergklinik und Klinik Saale, Reha-Zentrum, Bad Kissingen
}

\section{Kurz wirksame Insuline}

$\nabla$

Bei allen subkutan injizierten kurz wirksamen Insulinen ist eine im Vergleich zur körpereigenen Insulinsekretion abweichende Kinetik unvermeidbar. Dies gilt insbesondere für das gentechnologisch hergestellte Humaninsulin (Normalinsulin). Es liegt im subkutanen Fettdepot in Form von Hexameren vor, die sich für den Übertritt in die Blutbahn erst auflösen müssen. Durch einen Spritz-Ess-Abstand von ca. 15-30 Minuten kann das Wirkmaximum gegebenenfalls auf den Zeitpunkt der maximalen postprandialen Blutzuckerkonzentration ausgerichtet werden. Jedoch bleibt das Problem einer deutlich flacheren Wirkungskurve als bei der körpereigenen Insulinsekretion. Mögliche Folgen sind erhöhte postprandiale Blutzuckerspitzen und/oder Hypoglykämien durch eine längere Insulinwirkung als die mahlzeitenbedingte Glukosebelastung. Mit Zwischenmahlzeiten kann der HypoglykämieNeigung zwar begegnet werden, jedoch können die erneute Glukosebelastung, die zusätzliche Kalorienzufuhr und der fixe Zeitpunkt der zusätzlichen Nahrungsaufnahme von Nachteil sein.

Bei den drei kurz wirksamen Insulinanaloga Insulin lispro (Humalog ${ }^{\circledR}$, Liprolog ${ }^{\circledR}$ ), Insulin aspart $\left(\right.$ NovoRapid $^{\circledR}$ ) und Insulin glulisin (Apidra ${ }^{\circledR}$ ) ist die Neigung zur Ausbildung von Hexameren herabgesetzt, indem das Humaninsulinmolekül in der BKette an einer oder zwei Positionen verändert wurde (Insulin lispro: die Aminosäuren 28 (Lysin) und 29 (Prolin) liegen in umgekehrter Reihenfolge vor, Insulin aspart: Asparaginsäure statt Prolin an Position 28, Insulin glulisin: Glutaminsäure statt Lysin an Position 29, Lysin statt Asparagin an Position 3 [5]. Dadurch tritt die Insulinwirkung innerhalb von 5-15 Minuten nach subkutaner Injektion ein, so dass in der Regel kein Spritz-Ess-Abstand notwendig ist [13]. Dies kann vorteilhaft sein, wenn der Zeitpunkt für eine Mahlzeit schwer vorhersehbar ist. Einzelne Patientengruppen können aber von dem Wirkprofil des Humaninsulins profitieren (z.B. bei Gastroparese). Ein kurzer SpritzEss-Abstand kann auch bei den kurz wirksamen Insulinanaloga sinnvoll sein, wenn Mahlzeiten mit hoher glykämischer Last auf eine verstärkte Insulinresistenz treffen (z.B. in den Morgenstunden).

Die maximale Serumkonzentration der kurz wirksamen Insulinanaloga ist nach subkutaner Gabe deutlich höher und die Wirkungsdauer kürzer als bei kurz wirksamem Humaninsulin ( Abb.1) [13]. Dementsprechend zeigen kurz wirksame Insulinanaloga eine signifikant bessere postprandiale Glukoseregulation $[13,21,30]$, bei gleichzeitig besserer Hemmung der Glukoseproduktion in der Leber [2].

In großen epidemiologischen Untersuchungen gab es Anzeichen für kardiovaskuläre Risiken durch erhöhte postprandiale Blutzuckerwerte [24]. Kleinere Studien weisen darauf hin, dass die effektivere Senkung postprandialer Blutzuckerspitzen durch kurz wirksame Insulinanaloga im Vergleich zu Humaninsulin kardiovaskuläre Parameter - wie z.B. Endothelfunktion, postprandiale Myokarddurchblutung und diastolische Herzfunktion - verbessern $[10,32,33]$. Retrospektive Datenbankanalysen aus deutschen Arztpraxen ergaben reduzierte makrovaskuläre Ereignishäufigkeiten für Insulinanaloga im Vergleich zu Humaninsulin [16, 27].

\section{kurzgefasst}

Bei kurz wirksamen Insulinanaloga ist in der Regel kein Spritz-Ess-Abstand notwendig und die postprandiale Glukoseregulation gegenüber Humaninsulin verbessert.

\section{Basalinsuline}

$\nabla$

Bei herkömmlichen Basalinsulinen wird der Verzögerungseffekt durch eine Bindung an das neutrale Protamin Hagedorn (NPH) erreicht. NPH-In-

\section{Diabetologie}

Schliusselwörter

$\checkmark$ Diabetes

OHumaninsulin

Insulinanaloga

$\nabla \mathrm{HbA}_{1 \mathrm{c}}$

Hypoglykämien

Körpergewicht

Keywords

diabetes

Duman insulin

insulin analogues

D $\mathrm{HbA}_{1 \mathrm{c}}$

hypoglycemia

$\checkmark$ body weight eingereicht 20.12 .2013 akzeptiert 10.04.2014

\section{Bibliografie}

DOI 10.1055/s-0034-1387263 Dtsch Med Wochenschr 2014; 139: 2199-2203 - (c) Georg Thieme Verlag KG · Stuttgart . New York · ISSN 0012-0472

Korrespondenz Prof. Dr. med. Baptist Gallwitz Medizinische Klinik IV Universitätsklinikum Tübingen Otfried-Müller-Str. 10 72076 Tübingen Tel. 07071/298-2093 eMail baptist.gallwitz@ med.uni-tuebingen.de 


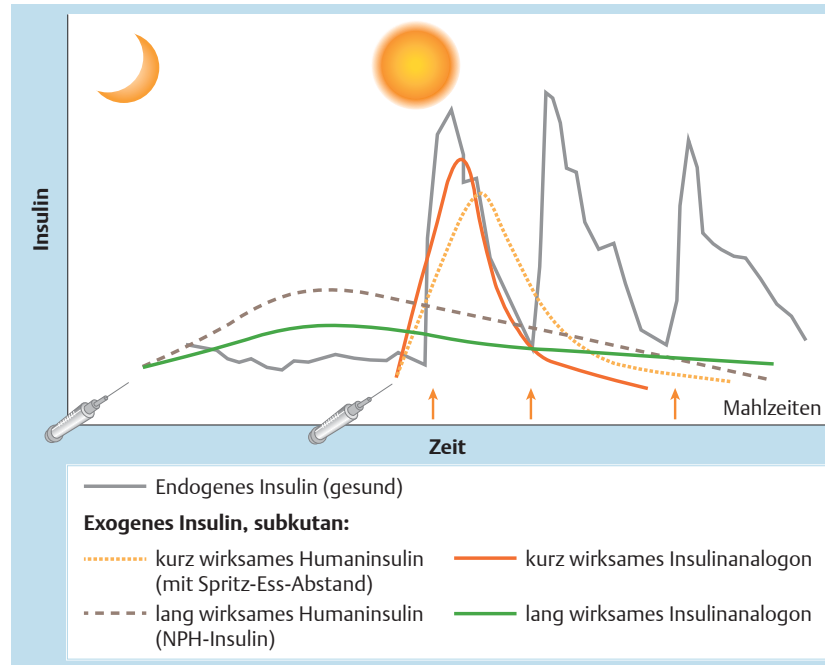

Abb.1 Wirkprofile der kurz und lang wirksamen Insuline nach subkutaner Injektion - schematische Darstellung (Darstellung auf Basis von Daten aus [25]).

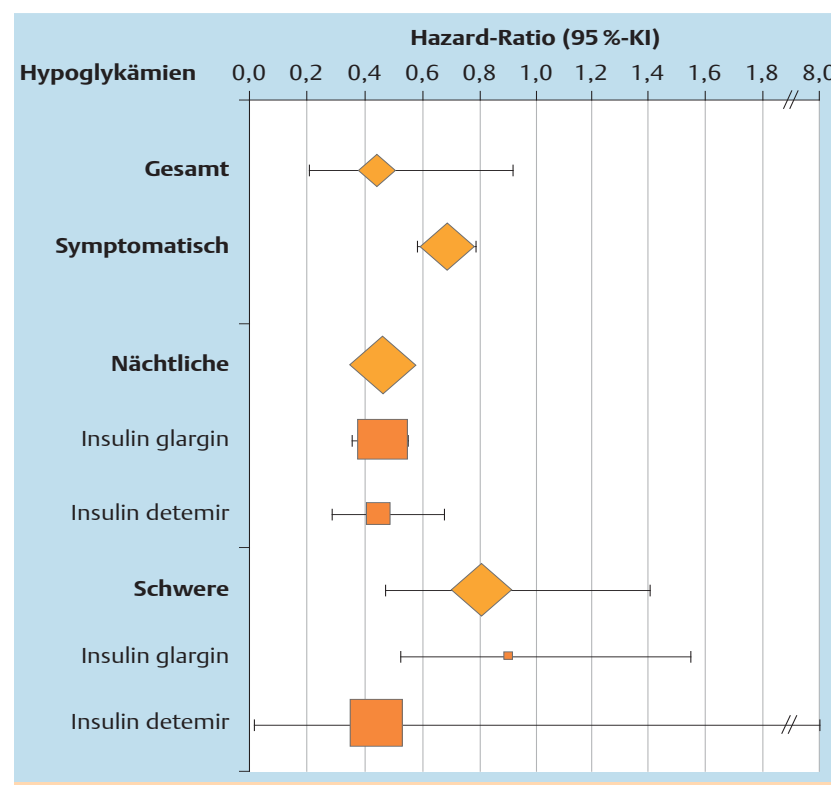

Abb.2 Metaanalyse zu Hypoglykämien bei lang wirksamen Insulinanaloga im Vergleich zu NPH-Insulin bei Typ-2-Diabetes [22]. Dargestellt ist das Risikoverhältnis (Hazard-Ratio mit 95\%-Konfidenzintervall [KI]) für die Inzidenz von Hypoglykämien (gesamt, symptomatisch, nächtliche, schwere) unter Verwendung lang wirksamer Insulinanaloga im Vergleich zu NPH-Insulin. Das Risiko für Hypoglykämien gesamt, sowie für symptomatische und nächtliche Hypoglykämien ist unter lang wirksamen Insulinanaloga signifikant geringer im Vergleich zu NPH-Insulin.

sulin hat den Nachteil, dass das gründliche Durchmischen der Suspension zur Injektion zeitaufwendig und fehleranfällig ist [1]. Außerdem ist das Wirkmaximum nach 4-10 Stunden relativ stark ausgeprägt, wodurch insbesondere in der Nacht aufgrund der dann erhöhten Insulinempfindlichkeit Hypoglykämien auftreten können. Bei den lang wirksamen Insulinanaloga Insulin glargin (Lantus $^{\circledR}$ ) und Insulin detemir (Levemir ${ }^{\circledR}$ ) ist ein Durchmischen nicht notwendig, beides sind klare Lösungen.

Der Verzögerungseffekt von Insulin glargin beruht darauf, dass es durch geringfügige Veränderungen am Humaninsulin-Molekül (A-Kette: Glycin anstelle von Asparagin an Position 21; BKette: zusätzlich zwei Arginine an Position 31 und 32) nur in saurem Milieu löslich ist und im Unterhautfettgewebe Mikrokristalle bildet [5]. Diese lösen sich langsam auf und setzen die Insulinmonomere frei.

Bei Insulin detemir ist nach Abspaltung einer terminalen Aminosäure eine Fettsäure gebunden, sodass sich im subkutanen Fettgewebe Dihexamere bilden. Sie zerfallen langsam in Monomere [5]. Durch die Fettsäure wird Insulin detemir nach Injektion an Albumin gebunden. Dies bedingt möglicherweise eine gleichmäßigere Freisetzung von Insulin detemir und könnte die geringere intraindividuelle Variabilität des Wirkprofils von Insulin detemir im Vergleich zu NPH-Insulin, aber auch im Vergleich zu Insulin glargin erklären $[7,15]$.

Im Vergleich zu NPH-Insulin ist bei lang wirksamen Insulinanaloga das Wirkmaximum reduziert und die Wirkdauer deutlich verlängert ( Abb.1) [5]. Dabei kann die Wirkdauer der Insuline individuell und dosisabhängig unterschiedlich sein.

Während NPH-Insulin aufgrund seines kürzeren Wirkprofils üblicherweise zur Nacht gegeben wird, können lang wirksame Insulinanaloga zu jeder Tageszeit verabreicht werden. Der Zeitpunkt sollte dann allerdings von Tag zu Tag beibehalten werden. Für die abendliche Gabe spricht die bessere Wirkung auf den Nüchternblutzucker, während sich bei morgendlicher Gabe die Blutzuckerwerte vor dem Abendessen besser darstellen. Vorrangig benötigen Patienten mit Typ-2-Diabetes das Basalinsulin zur Nacht, um der Glukoseneubildung der Leber zu begegnen. Ein eventueller Bedarf über Tag lässt sich durch ansteigende Blutzuckerspiegel im Rahmen eines Fastentests erfassen.

Metaanalysen von Studien zu Insulin detemir oder Insulin glargin versus NPH-Insulin ergaben bei vergleichbarem $\mathrm{HbA}_{1 \mathrm{c}}$ signifikante Reduktionen der symptomatischen und der nächtlichen Hypoglykämien [22, 35] ( Abb.2). Außerdem zeigte sich unter Insulin detemir, nicht jedoch unter Insulin glargin, eine signifikant günstigere Entwicklung des Körpergewichts [22].

\section{kurzgefasst}

Ein Durchmischen der Suspension ist nur bei NPH-Insulinen notwendig. Lang wirksame Insulinanaloga sind aufgrund der größeren Flexibilität bezüglich des Injektionszeitpunkts und des geringeren Hypoglykämie-Risikos von Vorteil.

\section{Lang wirksame Insulinanaloga im Vergleich \\ $\checkmark$}

Die Wirkdauer von Insulin detemir und Insulin glargin beträgt bei der Therapie des Typ-2-Diabetes mit Dosierungen von 0,4-0,8 E/kg KG bis zu 24 Stunden $[7,19]$. Hinsichtlich Wirksamkeit und Hypoglykämie-Risiko ergab sich in einer Gesamtauswertung von Studien kein klinisch relevanter Unterschied zwischen Insulin detemir und Insulin glargin bei Patienten mit Typ-2-Diabetes [34]. Insulin detemir war mit einer geringeren Gewichtszunahme assoziiert als Insulin glargin, aber auch mit einer höheren Insulindosis [34]. Allerdings wurde Insulin detemir in diesen Studien häufig zweimal täglich gegeben. Explorative Studienanalysen zum Typ-2-Diabetes zeigen jedoch, dass die zweimal tägliche Gabe von Insulin detemir im Vergleich zur einmal täglichen Gabe zu höheren Dosen führte und den Gewichtsvorteil verringerte ohne die Blutzuckerkontrolle weiter zu verbessern [15]. 
Im Januar 2013 wurde Insulin degludec (Tresiba ${ }^{\circledR}$ ) von der europäischen Arzneimittelbehörde neu zugelassen. Im Vergleich zu Insulin glargin ist seine Halbwertszeit doppelt so lang mit einer signifikant geringeren intraindividuellen Variabilität der blutzuckersenkenden Wirkung von Tag zu Tag [4]. Die pharmakodynamischen Eigenschaften scheinen sich insbesondere auf das Risiko nächtlicher Hypoglykämien positiv auszuwirken. Die Rate an Hypoglykämien (gesamt, nächtlich) bei Typ-2-Diabetes war in der Metaanalyse signifikant niedriger im Vergleich zu Insulin glargin [28]. Insulin degludec kann bei Bedarf tageszeitlich flexibel verabreicht werden, wobei mindestens 8 und höchstens 40 Stunden Abstand zwischen zwei Injektionen einzuhalten sind [4].

\section{kurzgefasst}

Insulin detemir und Insulin glargin sind hinsichtlich Blutzuckereinstellung und Hypoglykämie-Risiko vergleichbar, wobei sich Insulin detemir vorteilhaft auf die Gewichtsentwicklung auswirken kann. Unter Insulin degludec war die Hypoglykämie-Inzidenz bei Typ-2-Diabetes noch niedriger als unter Insulin glargin.

\section{Mischinsuline}

$\nabla$

Misch- oder auch Kombinationsinsuline enthalten einen kurz und einen lang wirksamen Insulinbestandteil. Der lang wirksame Anteil besteht bei den derzeit auf dem Markt befindlichen Präparaten aus Protamin-gebundenem Insulinanalogon oder Humaninsulin, so dass diese deshalb auch vor Verwendung sorgfältig durchmischt werden müssen. Metaanalysen mit dem biphasischen Insulin aspart 30 ergaben im Vergleich zu humanem Mischinsulin einen signifikant geringeren postprandialen Blutzuckeranstieg bei vergleichbaren $\mathrm{HbA}_{1 \mathrm{c}}$-Reduktionen und vergleichbaren Hypoglykämie-Raten (gesamt) [3, 26].

\section{Reduktion des $\mathrm{HbA}_{1 \mathrm{c}}$}

Bei insulinnaiven Patienten mit unzureichend eingestelltem Typ2-Diabetes senken Insulinanaloga den $\mathrm{HbA}_{1 \mathrm{c}}$-Wert im Mittel um 1 bis 2 Prozentpunkte gegenüber der vorherigen Therapie. Hierzu stehen vier verschiedene Formen der Insulintherapie (und ihre Abwandlungen) zur Verfügung:

- Basal unterstützte orale Therapie (BOT), bei der ergänzend zum oralen Antidiabetikum ein Basalinsulin injiziert wird [6, 8, 12,20].

- Supplementäre Insulintherapie (SIT), bei der ein kurz wirksames Insulin zu den Hauptmahlzeiten injiziert wird, eventuell in Kombination mit Metformin und bei Bedarf ergänzt durch Basalinsulin $[12,14,18]$.

- Konventionelle Insulintherapie (CT) mit Mischinsulin mit oder ohne orales Antidiabetikum [3, 12, 14, 26].

- Intensivierte Insulintherapie (ICT) mit kurz wirksamem Insulin und Basalinsulin [17].

In einer randomisierten klinischen Studie mit Insulinanaloga bei insulinnaiven Patienten mit Typ-2-Diabetes schnitt die BOT hinsichtlich der $\mathrm{HbA}_{1 \mathrm{c}}$-Reduktion signifikant schlechter ab als die supplementäre und die konventionelle Insulintherapie [12]. Dagegen war die BOT mit der geringsten Hypoglykämierate und der günstigsten Gewichtsentwicklung assoziiert. Nach dem ersten Studienjahr musste bei einem Großteil der Patienten ein weiteres Insulin eingesetzt werden, um den Zielwert zu erreichen (konventionelle Insulintherapie 67,7\%, supplementäre Insulintherapie 73,6\%, BOT
81,6\%). Zum Studienende nach 3 Jahren war die $\mathrm{HbA}_{1 \mathrm{c}}$-Reduktion bei den drei Gruppen vergleichbar [11]. Auch in deutschen Arztpraxen zeigte sich, dass mehr als $80 \%$ der zunächst mit einer supplementären Insulintherapie behandelten Patienten im ersten Jahr zusätzlich ein Basalinsulin benötigten [29]. Daher sollte nach Beginn einer BOT oder supplementären Insulintherapie auf eine bedarfsgerechte zeitnahe Intensivierung der Therapie geachtet werden. Bei bereits laufender Therapie mit herkömmlichen Insulinen kann nach Umstellung auf Insulinanaloga der $\mathrm{HbA}_{1 \mathrm{c}}$-Wert zusätzlich reduziert werden. Dabei können durchschnittliche Reduktionen um 1 bis 2 Prozentpunkte erreicht werden [9, 14].

In randomisierten klinischen Studien zu Insulinen werden für die Behandlungsgruppen üblicherweise identische Blutzuckerzielbereiche und damit ähnliche $\mathrm{HbA}_{1 \mathrm{c}}$-Werte am Studienende angestrebt, um eine Vergleichbarkeit insbesondere hinsichtlich der Hypoglykämieraten zu erreichen. Dadurch wurden in diesen Studien in der Regel vergleichbare Reduktionen des $\mathrm{HbA}_{1 \mathrm{c}^{-}}$ Werts unter Insulinanaloga und herkömmlichen Insulinen festgestellt $[22,30]$.

\section{kurzgefasst}

Bei insulinnaiven Patienten reduziert die Insulintherapie den $\mathrm{HbA}_{1 \mathrm{c}}$-Wert im Mittel um 1 bis 2 Prozentpunkte. Bei unzureichend eingestelltem $\mathrm{HbA}_{1 \mathrm{c}}$ unter Humaninsulin können Insulinanaloga eine zusätzliche Reduktion bewirken.

\section{Einfluss der Insulintherapie auf verschiedene Parameter}

\section{Hypoglykämien}

Die Angst vor Hypoglykämien ist ein wesentlicher Hinderungsgrund für den Beginn einer Insulintherapie [17]. In einer Beobachtungsstudie kam es nach Beginn der Insulintherapie mit Insulinanaloga nur bei der intensivierten Insulintherapie zu einem signifikanten Anstieg der Hypoglykämien, nicht jedoch bei der BOT, supplementären oder konventionelle Insulintherapie [14]. Bei einer BOT ist in den ersten 12 Wochen lediglich bei einem von 500 Patienten mit einer schweren Hypoglykämie und bei einem von 50 Patienten mit einer symptomatischen Hypoglykämie zu rechnen [6]. Gleichwohl kann es bei älteren insulinnaiven Patienten über 65 Jahre nach Einleitung einer BOT zu vermehrten Hypoglykämien kommen [20].

\section{Blutglukoseschwankungen}

Bei schwankenden Blutzuckerwerten mit Hypo- und Hyperglykämien sollten auch Lipohypertrophien als Ursache in Betracht gezogen werden. Diese gutartigen Fettgewebsveränderungen führen an den Spritzstellen zu Bereichen mit stark unterschiedlicher regionaler Durchblutung und Resorptionsfähigkeit [36]. Daher sollte auf die richtige Spritztechnik und eine Rotation der Injektionsstellen geachtet werden. Außerdem sind die Bildung einer Hautfalte, eine langsame Injektion, die richtige Auswahl des Injektionsorts und der Nadelwechsel im Zusammenhang mit der Insulingabe zu beachten. Gemäß einer Erhebung aus Deutschland traten bei $42 \%$ der Patienten mit Diabetes unerklärliche Blutglukoseschwankungen auf, die durch ungünstige oder falsche Injektionsgewohnheiten bzw. -auffälligkeiten verursacht gewesen sein könnten [31]. 


\section{Körpergewicht}

Nach Einleitung einer Insulintherapie kann das Körpergewicht ansteigen. Als Ursachen kommen insbesondere die verbesserte Glukoseverwertung, eine Appetitsteigerung und bei Humaninsulin eventuell notwendige Zwischenmahlzeiten in Betracht. Vorteilhafte Ergebnisse zum Gewichtsproblem wurden vor allem bei einer BOT mit Insulin detemir und einer supplementären Insulintherapie mit kurz wirksamen Insulinanaloga festgestellt $[8,18]$.

\section{kurzgefasst}

Die Einleitung einer BOT, supplementären oder konventionellen Insulintherapie ist nicht zwangsläufig mit einem erhöhten Hypoglykämierisiko verbunden. Lipohypertrophien sind eine wichtige Ursache für unerklärliche Blutglukoseschwankungen. Der Gewichtsproblematik kann durch eine BOT mit Insulin detemir entgegengewirkt werden.

\section{Wechsel von herkömmlichen Insulinen auf Insulinanaloga}

Beim Wechsel von herkömmlichen Insulinen auf Insulinanaloga ist von Bedeutung, dass eine internationale Einheit Human- oder NPHInsulin einer Einheit des Insulinanalogons entspricht. Dies trifft auch für Mischinsuline zu. So kann die Umstellung in der Regel dosisgleich erfolgen. Gleichwohl sind Besonderheiten zu beachten:

- Ggf. ist eine Anpassung der Dosis des Mahlzeiteninsulins nötig.

- Bei Insulin glargin sollte die Tagesdosis um 20-30\% reduziert werden, wenn NPH-Insulin zuvor $2 \times$ täglich appliziert wurde.

- Erfahrungsgemäß benötigen Patienten mit $1 \times$ täglicher Injektion von Insulin detemir eine geringere Dosis als bei $2 \times$ täglicher Injektion [15].

- Grundsätzlich ist eine Dosisreduktion aus Sicherheitsgründen zu erwägen, wenn Hypoglykämien in der Vorgeschichte bekannt sind.

- Umstellungen von einem Insulinpräparat auf ein anderes müssen aus Sicherheitsgründen von Blutzuckermessungen und individuellen Dosisanpassungen begleitet werden.

- Der Patient sollte über die unterschiedlichen Wirkverläufe und sich daraus ergebende Veränderungen (z.B. hinsichtlich Spritzzeitpunkt) aufgeklärt werden.

\section{Zusammenfassung}

\section{$\nabla$}

Bei allen Formen der Insulintherapie konnten diverse Vorteile für Insulinanaloga im Vergleich zu herkömmlichen Insulinen festgestellt werden. Dementsprechend gibt es viele Konstellationen, bei
Tab.1 Insulinanaloga im Vergleich zu herkömmlichen Insulinen bei Typ-2Diabetes.

\begin{tabular}{|c|c|}
\hline Insulintyp & Charakteristika \\
\hline $\begin{array}{l}\text { Kurz wirksame } \\
\text { Insulinanaloga }\end{array}$ & $\begin{array}{l}\text { - schnelleres Anfluten, höhere Peakwirkung, kürzere } \\
\text { Wirkdauer [13] } \\
\text { - in der Regel kein Spritz-Ess-Abstand und keine } \\
\text { Zwischenmahlzeiten erforderlich [13] } \\
\text { - bessere Senkung postprandialer Blutzuckerspiegel } \\
\text { [13, 21, 30] } \\
\text { - bei Umstellung von Humaninsulin Reduktion des } \mathrm{HbA}_{1 c^{-}} \\
\text {Wertes und der Hypoglykämien möglich [9] } \\
\text { - Hinweise auf Verbesserung kardiovaskulärer Parameter } \\
\text { und Folgekomplikationen [10, 16, 27, 32, 33] } \\
\text { - Gabe auch postprandial möglich (z.B. geriatrische } \\
\text { Patienten) [23] }\end{array}$ \\
\hline Lang wirksame & - flacheres Wirkprofil, längere Wirkdauer [5] \\
\hline Insulinanaloga & $\begin{array}{l}\text { - kein zeitaufwendiges und fehleranfälliges Durchmischen } \\
\text { - Injektion einmal täglich möglich, tageszeitlich flexibel* } \\
\text { - intraindividuelle Schwankungen geringer [7] } \\
\text { - weniger Hypoglykämien, insbesondere nachts [22, 35] } \\
\text { - geringere Gewichtszunahme bei Insulin detemir [22] }\end{array}$ \\
\hline
\end{tabular}

denen Insulinanaloga bevorzugt verordnet werden sollten. Dies sind z.B. Probleme mit dem Spritz-Ess-Abstand, Zwischenmahlzeiten (kurz wirksame Humaninsuline), dem Durchmischen der Suspension oder der Notwendigkeit zur mehrmals täglichen Gabe (NPH-Insulin). Auch der Wunsch, beim Spritz-Zeitpunkt flexibler zu sein, gehört dazu. Bei der glykämischen Einstellung sind Insulinanaloga von Vorteil, da sie die postprandialen Blutzuckerspiegel verbessern, ihre Wirkung genauer vorhersagbar ist, sie zu weniger Hypoglykämien führen und im Falle von Insulin determir der Einfluss auf das Körpergewicht günstiger ist. Für kurz wirksame Insulinanaloga gibt es Hinweise darauf, dass diese im Vergleich zu Humaninsulinen positive Effekte auf kardiovaskuläre Parameter und Folgekomplikationen haben könnten. Gleichwohl können manche Patienten besser mit einem herkömmlichen Insulin eingestellt werden, z.B. bei Gastroparese oder wenn durch das Essverhalten das verzögerte und flachere Wirkprofil von Humaninsulin von Vorteil ist. Tab.1 gibt eine Übersicht über die Charakteristika von kurz und lang wirksamen Insulinanaloga.

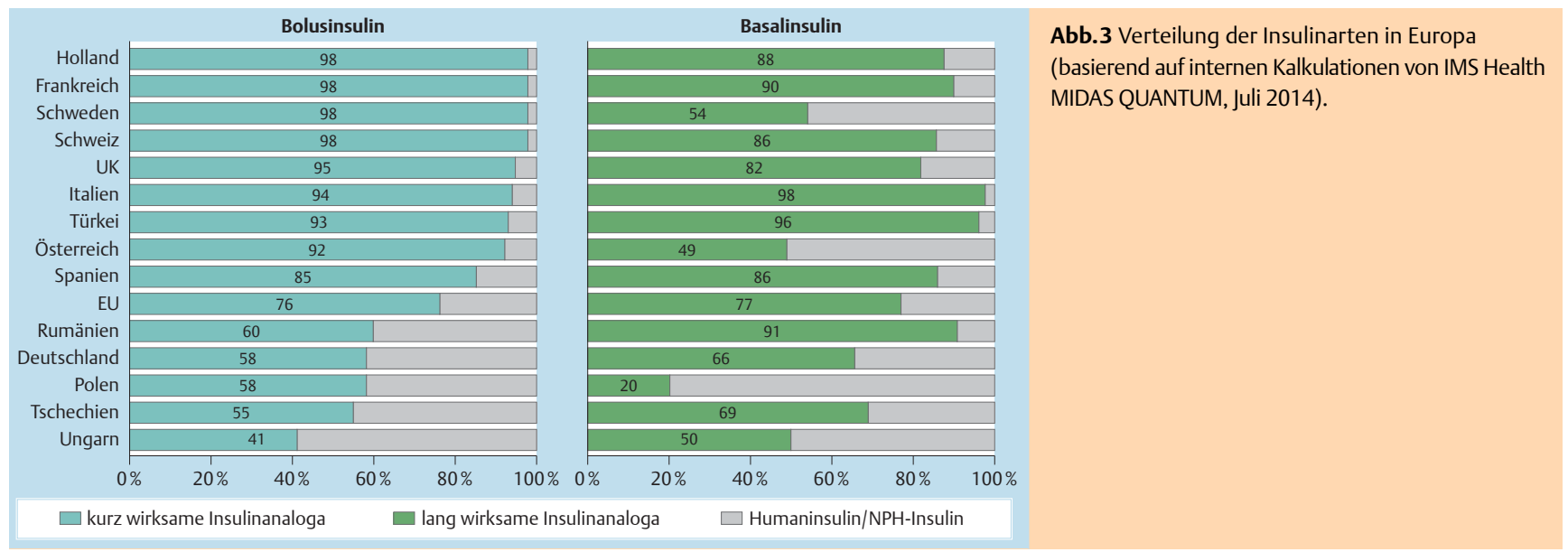


Unverändert fehlen große prospektive randomisierte Langzeitstudien zu den Insulinanaloga, die die Reduktion diabetesbezogener Komplikationen im Vergleich zu herkömmlichen Insulinen untersuchen. Dies führt zwangsläufig zu einer anderen Bewertung des Stellenwertes der Insulinanaloga, wenn insbesondere derartige Studien für die Analyse zugrunde gelegt werden. In anderen Ländern (wie z.B. Frankreich) erreicht der Verordnungsanteil der Insulinanaloga im Verhältnis zu herkömmlichen Insulinen bereits über 90\%, während dieser in Deutschland bei 58\% (Bolusinsulin) bzw. 66\% (Basalinsulin) liegt ( Abb.3).

\section{Konsequenz für Klinik und Praxis}

- Der Angst vor gehäuften Hypoglykämien und einer Gewichtszunahme kann mit der Auswahl einer geeigneten Insulintherapie bei Typ-2-Diabetes begegnet werden. - Nach Einleitung der Insulintherapie ist auf eine bedarfsgerechte zeitnahe weitere Intensivierung zu achten. - Bei der Wahl des Insulins sollten die Vorteile von Insulinanaloga bezüglich Praktikabilität, Blutzuckereinstellung und Hypoglykämie-Risiko berücksichtigt werden.

Autorenerklärung: B. G. führt Vortragstätigkeiten für Berlin-Chemie, Novo Nordisk, Lilly und Sanofi durch, ist Mitglied in Advisory Boards bei MSD, Novo Nordisk und Lilly und erhält Forschungsunterstützung von Novo Nordisk. G.-W. S. führt Vortragstätigkeiten für Novo Nordisk, Sanofi, Lilly und MSD durch und ist Mitglied verschiedener Advisory Boards. D. W. ist Mitarbeiterin der Novo Nordisk Pharma GmbH, Mainz, Abteilung Medical Affairs. Die Autoren danken Herrn Dr. med. Detlev Janssen für die redaktionelle Mitarbeit. Herr Janssen erklärt, dass seine Firma Med-iScene Concept $\mathrm{GmbH}$ diesbezüglich eine inhaltlich ungebundene Unterstützung von der Firma Novo Nordisk Pharma erhalten hat.

\section{Literatur}

1 Brown A, Steel JM, Duncan C et al. An assessment of the adequacy of suspension of insulin in pen injectors. Diabet Med 2004; 21: 604-608

2 Bruttomesso D, Pianta A, Mari A et al. Restoration of early rise in plasma insulin levels improves the glucose tolerance of type 2 diabetic patients. Diabetes 1999; 48: 99-105

3 Davidson JA, Liebl A, Christiansen JS et al. Risk of nocturnal hypoglycemia with biphasic insulin aspart 30 compared with biphasic human insulin 30 in adults with type 2 diabetes mellitus: a meta-analysis. Clin Ther 2009; 31: 1641-1651

4 Drab SR, Philis-Tsimikas A. A new option for glycemic control: insulin degludec, a new-generation basal insulin with an ultralong duration of action. Pharmacotherapy 2014; 34: 291-302

5 Evans M, Schumm-Draeger PM, Vora J et al. A review of modern insulin analogue pharmacokinetic and pharmacodynamics profiles in type 2 diabetes improvements and limitations. Diabetes Obes Metab 2011; 13: 677-684

6 Hanefeld M, Fleischmann H, Landgraf $W$ et al. EARLY-Studie: Frühe Basalinsulintherapie unter realen Bedingungen bei Typ-2-Diabetikern. Diabetes, Stoffwechsel und Herz 2012; 21: 91-97

7 Heise T, Pieber TR. Towards peakless, reproducible and long-acting insulins. An assessment of the basal analogues based on isoglycaemic clamp studies. Diabetes Obes Metab 2007; 9: 648-659

8 Hermanns $N$, Kaiser $M$, Reuter $H-M$ et al. Therapiezufriedenheit nach Beginn einer Insulintherapie bei Typ 2 Diabetes: DE-LIGHT. Diabetes, Stoffwechsel und Herz 2014; 23: 9-16

9 Hermansen K, Dornhorst A, Sreenan S. Observational, open-label study of type 1 and type 2 diabetes patients switching from human insulin to insulin analogue basal-bolus regimens: insights from the PREDICTIVE study. Curr Med Res Opin 2009; 25: 2601-2608

10 Hohberg $C$, Forst T, Larbig $M$ et al. Effect of insulin glulisin on microvascular blood flow and endothelial function in postprandial state. Diabetes Care 2008; 31: 1021-1025

11 Holman RR, Farmer AJ, Davies MJ et al. Three-year efficacy of complex insulin regimens in type-2-diabetes. N Engl J Med 2009; 361: 1736-1747

12 Holman RR, Thorne KI, Farmer AJ et al. Addition of biphasic, prandial, or basal insulin to oral therapy in type 2 diabetes. N Engl J Med 2007; 357 1716-1730
13 Home PD. The pharmacokinetics and pharmacodynamics of rapid-acting insulin analogues and their clinical consequences. Diabetes Obes Metab 2012; 14: 780-788

14 Home $P$, Naggar NE, Khamseh $M$ et al. An observational non-interventional study of people with diabetes beginning or changed to insulin analogue therapy in non-Western countries: the A1chieve study. Diabetes Res Clin Pract 2011; 94: 352-363

15 Keating GM. Insulin detemir: a review of its use in the management of diabetes mellitus. Drugs 2012; 72: 2255-2287

16 Kress $S$, Kostev K, Dippel FW et al. Micro- and macrovascular outcomes in Type 2 diabetic patients treated with insulin glulisine or human regular insulin: a retrospective database analysis. Int J Clin Pharmacol Ther 2012; 50: 821-829

17 Lau ANC, Tang T, Halapy $\mathrm{H}$ et al. Initiating insulin in patients with type 2 diabetes. CMAJ 2012; 184: 767-776

18 Lundershausen $R$, Potthoff $F$, Kaiser $M$ et al. Supplementäre Insulintherapie bei Patienten mit Typ-2-Diabetes - die PHAZIT ${ }^{\circledR}$-Studie. Diabetologie 2006; $1: 54-59$

19 Luzio SD, Dunseath GJ, Atkinson MD et al. A comparison of the pharmacodynamic profiles of insulin detemir and insulin glargine: A single dose clamp study in people with type 2 diabetes. Diabetes Metab 2013; 39: 537-542

20 Malek R, Gonzalez-Galvez G, El Naggar $N$ et al. Safety and effectiveness of insulin detemir in different age-groups in the A1chieve study. Diabetes Ther 2013; 4: 77-90

21 Mannucci E, Monami M, Marchionni N. Short-acting insulin analogues vs. regular human insulin in type 2 diabetes: a meta-analysis. Diabetes Obes Metab 2009; 11: 53-59

22 Monami M, Marchionni N, Mannucci E. Long-acting insulin analogues versus NPH human insulin in type 2 diabetes. A meta-analysis. Diabetes Res Clin Pract 2008; 81: 184-189

23 Ober SK, Watts S, Lawrence RH. Insulin use in elderly diabetic patients. Clinical Interventions in Aging 2006; 1: 107-113

24 Peter $R$, Okoseime OE, Rees A et al. Postprandial glucose - a potential therapeutic target to reduce cardiovascular mortality. Curr Vasc Pharmaco 2009; 7: 68-74

25 Polonsky KS, Given BD, van Cauter E. Twenty-four-hour profiles and pulsatile patterns of insulin secretion in normal and obese subjects. J Clin Invest 1988; 81: 442-448

26 Qayyum R, Bolen S, Maruthur $N$ et al. Systematic review: comparative effectiveness and safety of premixed insulin analogues in type 2 diabetes. Ann Intern Med 2008; 149: 549-559

27 Rathmann W, Kostev K. Lower incidence of recorded cardiovascular outcomes in patients with type 2 diabetes using insulin aspart vs. those on human regular insulin: observational evidence from general practices. Diabetes, Obesity and Metabolism 2013; 15: 358-563

28 Ratner R, Gough SCL, Mathieu C et al. Hypoglycaemia risk with insulin degludec compared with insulin glargine in type 2 and type 1 diabetes: a pre-planned meta-analysis of Phase 3 trials. Diabetes, Obesity and Metabolism 2013; 15: 175-184

29 Rendschmidt T, Wizemann E, Milek K et al. Einstieg in die supplementäre Insulintherapie zur Intensivierung der Behandlung des Typ 2 Diabetes Daten zu Insulindosistitration und Blutzuckermonitoring aus der SIT-Studie. Diabetologie 2008; 3: A214

30 Rys P, Pankiewicz O, Lach K et al. Efficacy and safety comparison of rapidacting insulin aspart and regular human insulin in the treatment of type 1 and type 2 diabetes mellitus: a systematic review. Diabetes Metab 2011: 37: 190-200

31 Schmeisl GW, Drobinski E. Koinzidenzen: Injektionsgewohnheiten, Lipohypertrophien, Glukoseschwankungen. Diabetes, Stoffwechsel und Herz 2009; 18: 251-258

32 Scognamiglio R, Negut C, de Kreutzenberg SV et al. Effects of different insulin regimes on postprandial myocardial perfusion defects in type 2 diabetic patients. Diabetes Care 2006; 29: 95-100

33 Siegmund $T$, von Bibra $H$, Riemer $M$ et al. Analogue versus human insulin therapy improves postmeal glucose and cardiac function in patients with type 2 diabetes with intensive conventional insulin therapy. Diabetologia 2009; 52 (Suppl. 1): S480

34 Swinnen SG, Simon ACR, Holleman $F$ et al. Insulin detemir versus insulin glargine for type 2 diabetes mellitusCochrane Database Syst Rev 2011; 6 : CD006383.10.1002/14651858.CD006383.pub2

35 Tschritter 0 , Fritsche A, Gallwitz B et al. Langwirkende Insulinanaloga in der Therapie des Diabetes mellitus Typ 1 und Typ 2. Eine Zusammenfassung der klinischen Studien und Metaanalysen. Diabetes und Stoffwechsel 2005; 14: 375-382

36 Vardar B, Kizilci S. Incidence of lipohypertrophy in diabetic patients and a study of influencing factors. Diabetes Res Clin Pract 2007; 77: 231-236 